\title{
How to ensure uninterrupted treatment of radiotherapy patients during delta variants of SARS-CoV-2 virus epidemic: the latest experience from Xi'an, China
}

\author{
Yongkai Lu' ${ }^{1} \cdot$ Ruijuan $\mathrm{Chen}^{2} \cdot \mathrm{Yi} \mathrm{Li}^{1}$
}

Received: 29 January 2022 / Accepted: 9 February 2022 / Published online: 23 February 2022

(c) The Author(s), under exclusive licence to Springer Science+Business Media, LLC, part of Springer Nature 2022

\begin{abstract}
This letter is some reflections on the articles by Lee et al. that have been published in MEDICAL ONCOLOGY. In this article, the experience of transferring, screening, and uninterrupted treatment of cancer patients in our radiotherapy center during Delta Variants of SARS-CoV-2 virus epidemic in Xi' an, China is presented in the form of a flowchart. By taking effective control measures, the radiotherapy center has not only effectively prevented the spread of the virus, but also ensured uninterrupted treatment for all patients. Therefore, we quickly share our center's experience so that more radiotherapy patients can benefit.
\end{abstract}

To the Editors

Numerous recent studies [1-3] have shown that interruption of radiation therapy in cancer patients during the COVID19 pandemic has a number of adverse effects. Therefore, we unreservedly support the proposal of Lee et al. [2] that the management of patients with suspected COVID-19 requires precise and systematic criteria that must also take into account the input of the radiation oncologist responsible for the patient. However, most investigators, including Lee et al. [2], have focused their research on risk factors for patients to discontinue radiotherapy and how to compensate patients for dose deviations caused by discontinuation of radiotherapy. Few people care about how to take measures to ensure that cancer patients try not to interrupt radiation therapy during the epidemic. Therefore, we would like to share the management experience of our radiotherapy center during the epidemic for the reference of colleagues in radiotherapy around the world.

Yongkai Lu

luyongkai@xjtufh.edu.cn

1 Department of Radiation Oncology, The First Affiliated Hospital of Xi' an Jiaotong University, No. 277, Yanta West Road, Xi' an 710061, Shaanxi, China

2 Xi' an Central Hospital, The Affiliated Hospital of Xi' an Jiaotong University, Xi' an 710003, Shaanxi, China
Recently, the global SARS-CoV-2 (Covid-19) pandemic has infected at least 290 million people and has caused fatalities of over 5.4 million (https://covid19.who.int/ (accessed on January 6, 2022). Moreover, numerous variants have rapidly evolved (https://www.who.int/en/activities/track ing-SARS-CoV-2-variants/). The delta variant is currently the most transmissible to date. It is the primary cause of increased hospitalization rates in Xi' an city and can cause break-through infections in vaccinated individuals. Up to now, Xi' an has a total of 2048 confirmed cases of Delta virus infection (accessed on January 7, 2022). Therefore, given the $\mathrm{Xi}$ 'an epidemic our radiotherapy center proposed a policy through consultation and cooperation with the government to maintain treatment of patients requiring radiotherapy while preventing and controlling the epidemic. Thus, we urgently want to share our management and control measures with radiotherapy centers to help more cancer patients.

Figure 1 illustrates our transportation and treatment measures for cancer patients during the Delta virus epidemic. Due to this measure, 243 of the 287 cancer patients requiring radiotherapy underwent their normal treatment courses (the ratio is $84.6 \%$ ). Due to regional restrictions, the 44 other patients temporarily received radiotherapy at designated hospitals in the corresponding area. We have achieved uninterrupted radiotherapy for all patients in this radiotherapy center. To effectively implement the above policies, four conditions need to be met. 
Fig. 1 Flowchart of medical treatment for cancer patients in Xi'an, China, during the epidemic

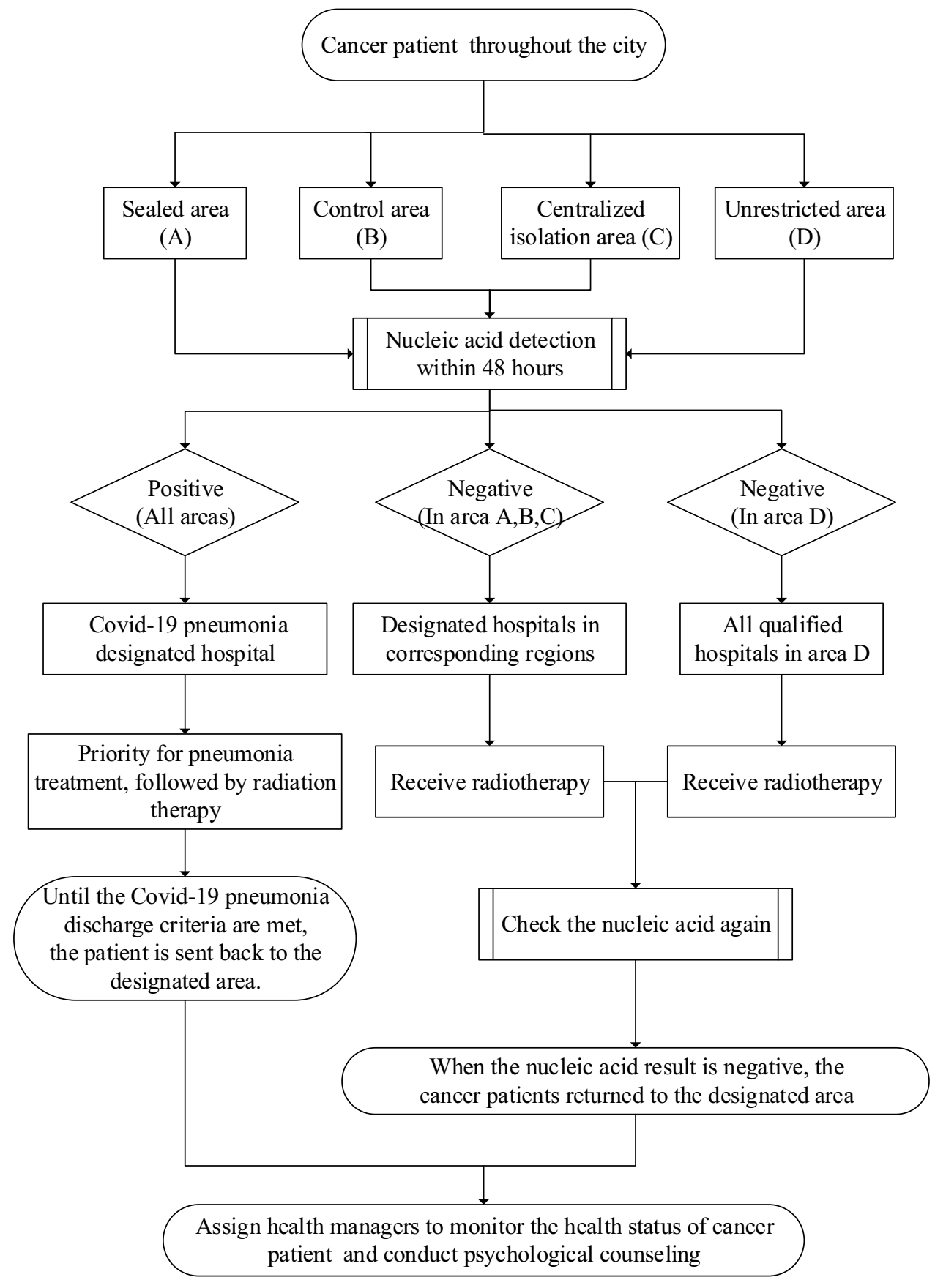

- Nucleic acid collection in the community or hospital once daily, and ensure that the results are available within 4-6 h.

- The public areas of the radiotherapy center are disinfected on time (once every half an hour), and the staff adopts secondary protection (wear disposable hats, N95 masks, protective masks, medical protective clothing, disposable gloves, and disposable shoes covers).

- All patients will make a telephone appointment to determine the treatment time. Each accelerator in the radiotherapy center only treats 4 patients per hour.
- Full cooperation with various departments related to epidemic prevention and control

In summary, we have provided a flow chart of the center's patient visits during the Delta variants of the SARS$\mathrm{CoV}-2$ virus epidemic. More cancer centers can consider our experience to help more cancer patients obtain timely and effective treatment interventions. 
Funding None.

\section{Declarations}

Conflict of interest The authors report no conflict of interest.

\section{References}

1. Maringe C, Spicer J, Morris M, et al. The impact of the COVID19 pandemic on cancer deaths due to delays in diagnosis in England, UK: a national, population-based, modelling study. Lancet Oncol. 2020;21(8):1023-34.
2. Lee S, Heo J. COVID-19 pandemic: a new cause of unplanned interruption of radiotherapy in breast cancer patients. Med Oncol. 2021;39(1):5.

3. Yu D, Hu W, Chen L, Fu Z, Song Q, Li X. Effect of radiotherapy interruption due to COVID-19 outbreak. Radiother Oncol. 2021;155:1-2.

Publisher's Note Springer Nature remains neutral with regard to jurisdictional claims in published maps and institutional affiliations. 